\title{
Reoperação em valvopatias: análise de 697 pacientes
}

\author{
Carlos Alberto Mussel BARROZO*, Rinaldo Costa SANTOS*, Cassio José SGARBI* Roberto Carin \\ LACANNA*, Neli Dalva MATHEUS, Elaine Moraes da SILVA*, Maria José Ricardo OLIVEIRA*, Maria \\ de Fátima F. Balthazar NEVES ${ }^{\star}$, Wilma Roberta ARDITO*, Roberto Hoshino KIOSHI*, Roberto Vito \\ ARDITO*
}

\section{RBCCV 44205-238}

BARROZO, C. A. M.; SANTOS, R. C.; SGARBI, C. J.; LACANNA, R. C.; MATHEUS, N. D.; SILVA, E. M.; OLIVEIRA, M. J. R.; NEVES, M. F. F. B.; ARDITO, W. R.; KIOSHI, R. H.; ARDITO, R. V. - Reoperação em valvopatias: análise de 697 casos. Rev. Bras. Cir. Cardiovasc., 9 (2): 109-112, 1994.

RESUMO: O estudo objetivou avaliar resultados imediatos e tardios da cirurgia das reoperaçōes em pacientes portadores de valvopatias, analisando o seguimento do paciente e nāo da prótese empregada. Foram analisados retrospectivamente 697 (419 fem. e 278 masc.) pacientes que foram reoperados entre 1970 a 1993. A idade média foi de $43,4 \pm 17,9$ anos. Como diagnóstico pré-operatório, $281(40,3 \%)$ apresentavam disfunção de prótese, $212(30,5 \%)$ reestenose pós comissurotomia, $103(14,7 \%)$ tiveram rotura e $101(14,5 \%)$ trombose da prótese. Reoperaçāo em valvopatias representou uma média de $7,38 \%$ de todo o movimento cirúrgico do Serviço, sendo, nos últimos 2 anos, $26,7 \%$ das operaçōes por valvopatia. A mortalidade hospitalar foi de $9,89 \%$, e o intervalo entre a $1^{2}$ e a $2^{2}$ operação foi de $77 \pm 37$ meses. Desses pacientes, 110 já foram submetidos a uma $3^{\text {a }}$ operação no nosso Serviço, com intervalo médio de $81 \pm 28$ meses e 10 já foram submetidos a uma $4^{\circ}$ operação. A mortalidade tardia foi de $16,6 \%$ e o seguimento médio de 14 anos, com perda de seguimento de $18,7 \%$ de pacientes. Ao contrário da maioria dos estudos de reoperaçōes onde é acompanhada a evolução de determinada prótese, nós procuramos enfocar o paciente e sua evolução clinica. Os resultados demonstram que a incidência de reoperaçōes na prática do Serviço vem aumentando sucessivamente e que esse tipo de operação pode ser realizado com um risco aceitável, e seus resultados, ainda que não excelentes a longo prazo, permitem ao paciente uma sobrevida razoável e a oportunidade de uma nova operação.

DESCRITORES: valvas cardiacas, cirurgia; próteses valvulares cardiacas, cirurgia; valvopatias, evolução de pacientes.

\section{INTRODUÇÃO}

A cirurgia de reoperação para tratamento das valvopatias é um procedimento que vem aumentando na prática dos serviços de cirurgia cardiaca, devido, principalmente, à baixa mortalidade cirúrgica do primeiro procedimento e ao número crescente de operações realizadas. O uso de biopróteses, que apresentam vida útil limitada $1,4,7-10,13,17$, trombose, o crescimento de panus, a necessidade premente de suspensão da anticoagulação ou falência estrutural das próteses mecânicas 4, 11, 19, 21; a endocardite bacteriana 3,18 e, ainda, a falha da técnica cirúrgica na primeira operação são os principais fatores associados ao tempo de evolução dos primeiros pacientes operados em razão de uma valvopatia, que representam, hoje, $26,7 \%$ do nosso movimento cirúrgico para tratamento de valvopatias, nos últimos 2 anos.

Analisamos a evolução dos nossos pacientes reoperados, independente do tipo, posiçäo ou técnica de substituição ou plastia da sua valva.

Trabalho realizado no Departamento de Cirurgia Cardiaca do IMC - Instituto de Moléstias Cardiovasculares, São José do Rio Preto, SP, Brasil. Apresentado ao $21^{2}$ Congresso Nacional de Cirurgia Cardiaca. Porto Alegre, RS, 24 a 26 de março, 1994.

- Do Instituto de Moléstias Cardiovasculares. São José do Rio Preto.

Endereço para correspondência: Roberto Vito Ardito. Rua Castelo D'Água 3030. CEP 15015-210 São José do Rio Preto, SP, Brasil. Fax.: [0172] 323764 
BARROZO, C. A. M.; SANTOS, R. C.; SGARBI, C. J.; LACANNA, R. C.; MATHEUS, N. D.; SILVA. E. M.; OLIVEIRA, M. J.R.; NEVES, M. F. F. B.; ARDITO, W. R.; KIOSHI, R. H.; ARDITO, R. V. - Reoperaçāo em valvopatias: análise de 697 casos. Rev. Bras. Cir. Cardiovasc., 9 (2): 109-112, 1994.

\section{CASUISTICA E MÉTODOS}

Utilizando o banco de dados do Departamento de Cirurgia Cardiaca do Instituto de Moléstias Cardiovasculares de São José do Rio Preto (IMC), juntamente com a análise individual do prontuário médico, foram avaliados, retrospectivamente, 697 pacientes submetidos a reoperações para o tratamento de uma ou mais valvopatia. O período de análise ficou compreendido entre setembro de 1970 e junho de 1993. Foram considerados para o estudo todos os pacientes que haviam sido submetidos a um primeiro procedimento cirúrgico para tratamento de lesão valvar, sendo excluidos os que necessitaram reoperação para correção de valvopatia, porém haviam sido submetidos a uma primeira operação não valvar.

A idade variou entre 13 e 72 anos, com a média de $43,4 \pm 17,9$ anos. Com $419(60,1 \%)$ pacientes femininos e $278(39,9 \%)$ masculinos.

Com diagnóstico pré-operatório, 281 (40,3\%) apresentavam disfunção de prótese, 212 (30,5\%) reestenose pós-comissurotomia, $103(14,7 \%)$ rotura da prótese e $101(14,5 \%)$ trombose da prótese.

\section{RESULTADOS}

Reoperaçāo em valvopatias representou uma média de $7,38 \%$ de todo o movimento cirúrgico do Serviço, sendo, nos últimos 2 anos, $26,7 \%$ das operaçōes de lesões valvares. A mortalidade hospitalar foi de $69(9,89 \%)$ pacientes com as causas descriminadas na Tabela 1.

A mortalidade tardia foi de $104(16,6 \%)$ pacientes sendo as principais causas relacionadas na Tabela 2.

O seguimento médio é de 14 anos, com perda de seguimento de $18,7 \%$ dos pacientes. O intervalo entre a $1^{a}$ e a $2^{a}$ operação foi de $77 \pm 37$ meses. Desses pacientes, 110 foram submetidos a uma $3^{a}$ operação, com a mortalidade hospitalar de 13 $(11,8 \%)$ pacientes e com intervalo médio de $81 \pm 28$

TABELA 1

\begin{tabular}{lc}
\hline CAUSAS DE OBITO HOSPITALAR & TOTAL $(\%)$ \\
\hline Síndrome de Baixo Débito & $24(34,8)$ \\
Insuficiência Respiratória & $13(18,8)$ \\
Septicemia & $9(13,1)$ \\
Insuficiência Renal & $8(11,6)$ \\
Hemorragia & $6(8,7)$ \\
Embolia Pulmonar & $5(7,2)$ \\
Outras & $4(5,8)$ \\
\hline Total & $69(100)$ \\
\hline
\end{tabular}

TABELA 2

\begin{tabular}{ll}
\hline CAUSAS DE ÓBITO TARDIO & TOTAL (\%) \\
\hline Insuficiência Cardiaca Congestiva & $29(27,8)$ \\
Edema Agudo de Pulmāo & $18(17,3)$ \\
Endocardite Bacteriana & $15(14,5)$ \\
Causas Nāo Cardiacas & $13(12,5)$ \\
Outras & $20(19,2)$ \\
Ignoradas & $9(8,7)$ \\
\hline Total & $104(100)$ \\
\hline
\end{tabular}

meses, e apenas 10 submetidos a uma $4^{3}$ operação, com 3 óbitos hospitalares.

\section{COMENTÁRIOS}

Reoperação em valvopatias apresenta um risco operatório ainda elevado e uma importante mortalidade tardia. Os fatores predisponentes a esses resultados são complexos e têm uma particularidade sócio-regional extremamente significativa e com variações complexas. Entretanto, com os dados apresentados, podemos realizar uma análise primária do assunto.

A mortalidade hospitalar com suas diversas causas (Tabela 1), está relacionada a fatores, como idade do paciente, procedimentos realizados previamente função ventricular pré-operatória, presença de endocardite e outras lesōes associadas, como coronariopatia e doenças pulmonares. $\mathrm{Na}$ nossa série encontramos uma mortalidade hospitalar comparável à de outros autores $2,12,15,16,22$. As principais causas de mortalidade também se mostram semelhantes, porém uma importante causa de morbimortalidade, que é a hemorragia incontrolável por acidente de reabertura $6,{ }^{14}$, não foi observada nos nossos pacientes; isso se deve, principalmente, à técnica de abertura do esterno com a serra oscilante, que é a rotina do nosso meio, diferindo do padrão internacional.

Com a melhoria das técnicas de circulação extracorpórea (CEC) e de proteção do miocárdio tivemos, nos últimos 2 anos, a mortalidade hospitalar de $5.45 \%$. Porém, acreditamos que a morbimortalidade ainda continuará alta por algum tempo, devido à gravidade dos nossos pacientes e ao número de sobreviventes da primeira reoperação, que retornarão, consecutivamente, para as próximas reintervenções.

Como já foi repetidamente dito, acreditamos que "a substituição de uma valva por uma prótese é a troca de uma doença pela outra". A evolução tardia dos pacientes é extremamente complexa e, 
BARROZO, C. A. M.; SANTOS, R. C.: SGARBI, C. J.; LACANNA, R. C.; MATHEUS, N. D.; SILVA, E. M.; OLIVEIRA, M. J. R.; NEVES, M. F. F. B.; ARDITO, W. R.; KIOSHI, R. H.; ARDITO, R. V. - Reoperaçāo em valvopatias: análise de 697 casos. Rev. Bras. Cir. Cardiovasc., 9 (2): 109-112, 1994.

no nosso grupo de pacientes estudados independentemente da prótese, posição ou técnica cirúrgica, o número de intercorrências e a necessidade de reintervenção foram elevadas, porém o intervalo entre as operações aumentou discretamente e sem uma alteração específica da mortalidade hospitalar, ao contrário dos dados da literatura 12, 22

Podemos concluir que, desde a introdução da substituição das valvas cardiacas 20 há mais de 3 décadas, milhares de vidas têm sido salvas e prolongadas, devido ao uso de próteses valvares; porém, as limitações e complicações associadas ainda impedem uma condição ideal de sobrevida para os pacientes portadores de valvopatias. Apesar do esforço para melhorar a qualidade das próteses e a técnica operatória, devemos acompanhar clinicamente o valvopata com a prevenção das possiveis complicações que possam deteriorar a sua condição clínica, que é fator primordial para um bom resultado de posteriores reintervençōes. $E$ mesmo que os resultados a longo prazo não sejam excelentes, os riscos da reoperação para o tratamento de valvopatias é aceitável e permite ao paciente, quando acompanhado de perto, uma sobrevida razoável e a possibilidade de uma nova intervenção.

BARROZO, C. A. M.; SANTOS, R. C.; SGARBI, C. J.; LACANNA, R. C.; MATHEUS, N. D.; SILVA. E. M.; OLIVEIRA, M. J. R.; NEVES, M. F. F. B.; ARDITO, W. R.; KIOSHI, R. H.; ARDITO, R. V. - Reoperations for valvopathies: analyzis of 697 patients. Rev. Bras. Cir. Cardiovasc., 9 (2): 109-112, 1994.

ABSTRACT: The aim of this study was to analyze the early and late results of the reoperation for valvar disease with the focus on the patient not on the prosthesis. A retrospective analysis of 697 patients $(419$ female, 278 male) was done between 1970 and 1990 . The mean age was $43.4+17.9$ years old. Pre-operative diagnosis were 281 (40.3\%) prosthesis dysfunction, (2/2) $30.5 \%$ restenosis pós-comissurotomy, $(103) 4,7 \%$ prosthesis rupture, $101(14.5 \%)$ prosthesis thrombosis. Reoperations for valvopathy were $7.38 \%$ of the total surgical procedures in our Service, and in the last 2 years $26.7 \%$ of the total valvar surgery procedures. The hospital mortality was $9.89 \%$, and the mean interval between the first and second procedure was $77+37$ months. A third operation was carry on 110 patients with the mean interval of $81+28$ and 10 had a fourth intervention. The late mortality was $16.6 \%$ and the mean follow up is 14 years with $18.7 \%$ lost of segment. Different from others studies where the prosthesis have been followed, our main goal was follow the patient's clinical evolution. The results shown that reoperations for valvopathy have increased in our Institution. The procedure can be done with an acceptable risk even though the long term results are not excellent the patients can have a reasonable survival and a chance of a new operation.

DESCRIPTORS: heart valves, surgery; heart valves, reoperation; valvopathies, follow-up of patients.

\section{REFERÊNCIAS BIBLIOGRÁFICAS}

1 AMATO, M. C. M.; PORMERANTZEFF, P.; GRIMBERG. M.; BELLOTTI, G.; PILLEGGI, F. - Evoluçāo imediata e tardia de pacientes com bioprótese porcina. Arq. Bras. Cardiol., 51: 381-384, 1988.

2 ANTUNES, M. J. - Isolated replacement of prosthesis on a bioprosthesis in the aortic valve position. Am. J. Cardiol., 59: 350-352, 1987.

3 ANTUNES, M. J. \& MAGALHĀES, M. P. - Isolated replacement of a prosthesis or a bioprosthesis in the mitral valve position. Am. J. Cardiol., 59: 346-349, 1987.
4 BORKON. A. M.: SOULE, L.; BAUGHMAN, K. L.; AUON, H.: GARDENER, T. J.; WATKINS Jr. L.; BAUMGARTENER, W. A.; GOTT, V. L.; REITZ, B. A. - Ten year analysis of the Bjork-Shiley standard aortic valves. Ann. Thorac. Surg., 43: 39-51, 1987.

5 BRAILE, D. M. - Prótese valvular de pericárdio bovino: desenvolvimento e aplicação clínica em posição mitral. Sāo Paulo, 1990 [Tese. Doutorado. Escola Paulista de Medicina].

6 CANVER, C. C.: MARRIN. C. A. S.: PLUME, S. K.; NUGENT, W. C. - Autologous pericardial flap for prevention of reentry injury in cardiac reoperations. Ann. Thorac. Surg., 55: 179-180, 1993. 
BARROZO, C. A. M.; SANTOS, R. C.; SGARBI, C. J.; LACANNA, R. C.; MATHEUS, N. D.; SILVA, E. M.; OLIVEIRA, M. J. R.; NEVES, M. F. F. B.; ARDITO, W. R.; KIOSHI, R. H.; ARDITO, R. V. - Reoperação em valvopatias: análise de 697 casos. Rev. Bras. Cir. Cardiovasc., 9 (2): 109-112, 1994.

CARVALHO, M. V. H.; VIEIRA, R. W.; SOUZA, J. M.; OLIVEIRA, S. A. - Desempenho tardio das biopróteses valvulares porcinas. Rev. Bras. Cir. Cardiovasc., 7: 28-37, 1992.

CRAVER, J. M.; WEINTRAUB, W. S.; JONES, E, L.; GUYTON, R. A.; HACTHER Jr., R. C. - Predictions of mortality, complication and length of stay in aortic valve replacement for aortic stenosis. Circulation, 78 (Supl. 1): 85-89, 1988.

9 FESSATIDIS, I.; HACKETT, D.; OAKLEY, C. M.; SAPSFORD, R. N.; BENTALL, H. H. - Ten-year clinical evaluation of isolated mitral valve and double valve replacement with Starr-Edward's prostheses. Ann. Thorac. Surg., 43: 368-372, 1987.

10

JONES, E. L.; WEINTRAUB, W. S.; CRAVER, J. M.; GUYTON, R. A.; COHEN, C. L.; CORRIGAN, V. E.; HACHTER Jr., C. R. - Ten year experience with the porcine bioprosthetic valve: interrelationship of valve survival and patient survival in 1,050 valve replacements. Ann. Thorac. Surg., 49: 370-384, 1990.

LINDBLOM, D. - Long-term clinical results after aortic valves replacement with the Bjork-Shiley prostheses. J. Thorac. Cardiovasc. Surg., 95: 658-667, 1988.

LYTLE, B. W.; COSGROVE, D. M.: TAYLOR, P. C.; GILL, C. C.; GOORMASTIC, M.; GOLDING, L. R.; STEWART, R. W.: LOOP, F. D. - Reoperations for valve surgery: perioperative mortality and determinants of risk for 1,000 patients, 1958-1984. Ann. Thorac. Surg., 42: 632-643, 1986.

MAGILLIGAN, D. J.; LEWIS Jr., J. W.; TILEY, B.; PETERSON, E. - The porcine bioprosthetic valve: twelve years later. J. Thorac. Cardiovasc. Surg., 89: 499-507, 1985.

MALM, T.; BOWALD, S.; BYLOCK, A.; BUSCH, C. Prevention of postoperative pericardial adhesions by closure of the pericardium with absorbable polymer patches: an experimental study. J. Thorac. Cardiovasc., Surg., 104: 600-607, 1992.
MASRI, Z.; GIRARDET, R.; ATTUM, A.; BARBIE, R.; YARED, I.; LANSING, A. - Reoperation for prosthetic heart valve dysfunction: 19 years experience. Texas Heart Inst. J., 17: 106-111, 1990.

PANSINI, S.: OTTINO, G.; FORSENNATI, P. G.; SERPIERI, G.; ZATTERA, G.; CASABONA, R.; DISUMA, M.; VILLANI, M.; POLETTI, G. A.; MOREA, M. - Reoperations on heart valve prostheses: an analysis of operative risks and late results. Ann. Thorac. Surg., 50: 590-596, 1990.

PELLETIER, L. C. - Valve replacement with bioprostheses for non-ischemic mitral insufficiency. Eur. Heart., J., 12 (Supl. B) 44-47, 1991.

18 SCOTT, W. C.; MILLER, D. C.; HAVERICH, A.; DAWKINS, K.; MITCHELL, R. S.; JAMIESON, S. W.; OYER, D. E.; STINSON, E. B.; BALDWIN, J. C.; SHUMWAY, N. E. - Determinants of operative mortality undergoing aortic valve replacement. J. Thorac. Cardiovasc. Surg., 89: 400-413, 1985.

SCHOEVARDTS, J. C.; BUCHE, M.; EL GARIANI, A.; LICHTSTEINER, M.; JAUMIM, P.; PONLOT, R.; CHALANT, CH-H - Twenty years experience with the model 6120 Starr-Edwards valve in the mitral position. J. Thorac. Cardiovasc. Surg., 94: 375-382, 1987.

STARR, A. \& EDWARDS, M. L. - Mitral replacement: clinical experiences with a ball-valve prostheses. Ann. Surg., 154: 726-731, 1961.

STEWART, S.; CIANCIOTTA, D.; HICKS, G. L.; DeWEESE, J. A. - The Lillehei-Kaster aortic valve prostheses. J. Thorac. Cardiovasc. Surg., 95: 1023$1030,1988$.

TEOH, K. T.; CHRISTAKIS, G. T.; WEISEL, R. D.; TONG, C. P.; MICKLEBOROUGH, L.; SCULLY, H. E.; GOLDMAN, B. E.; BAIRD, R. J. - Determinants of mortality and morbidity after multiple valve operations. Ann. Thorac. Surg., 43: 353-358, 1987. 